\title{
Cytosolic p120-catenin regulates growth of metastatic lobular carcinoma through Rock1-mediated anoikis resistance
}

\author{
Ron C.J. Schackmann, ${ }^{1}$ Miranda van Amersfoort,, ${ }^{1,2}$ Judith H.I. Haarhuis, ${ }^{1}$ Eva J. Vlug, ${ }^{3}$ \\ Vincentius A. Halim, ${ }^{1}$ Jeanine M.L. Roodhart, ${ }^{1}$ Joost S. Vermaat, ${ }^{1}$ Emile E. Voest, ${ }^{1}$ \\ Petra van der Groep, ${ }^{3,4}$ Paul J. van Diest, ${ }^{3}$ Jos Jonkers, ${ }^{5}$ and Patrick W.B. Derksen, ${ }^{1,2,3}$

\begin{abstract}
${ }^{1}$ Department of Medical Oncology, ${ }^{2}$ Cancer Center, ${ }^{3}$ Department of Pathology, and ${ }^{4}$ Division of Internal Medicine, University Medical Center Utrecht,
\end{abstract} \\ Utrecht, the Netherlands. ${ }^{5}$ Division of Molecular Biology, Netherlands Cancer Institute, Amsterdam, the Netherlands.
}

\begin{abstract}
Metastatic breast cancer is the major cause of cancer-related death among women in the Western world. Invasive carcinoma cells are able to counteract apoptotic signals in the absence of anchorage, enabling cell survival during invasion and dissemination. Although loss of E-cadherin is a cardinal event in the development and progression of invasive lobular carcinoma (ILC), little is known about the underlying mechanisms that govern these processes. Using a mouse model of human ILC, we show here that cytosolic p120-catenin (p120) regulates tumor growth upon loss of E-cadherin through the induction of anoikis resistance. $\mathrm{p} 120$ conferred anchorage independence by indirect activation of Rho/Rock signaling through interaction and inhibition of myosin phosphatase Rho-interacting protein (Mrip), an antagonist of Rho/Rock function. Consistent with these data, primary human ILC samples expressed hallmarks of active Rock signaling, and Rock controlled the anoikis resistance of human ILC cells. Thus, we have linked loss of E-cadherin - an initiating event in ILC development - to Rho/Rock-mediated control of anchorage-independent survival. Because activation of Rho and Rock are strongly linked to cancer progression and are susceptible to pharmacological inhibition, these insights may have clinical implications for the development of tailor-made intervention strategies to better treat invasive and metastatic lobular breast cancer.
\end{abstract}

\section{Introduction}

Breast cancer progression depends on the capacity of cells to invade, metastasize, and colonize distant sites. Loss of tumor cell adhesion and subsequent anchorage-independent survival are crucial steps in this process. In breast cancer, invasive ductal carcinoma (IDC) often coexpresses E-cadherin, $\beta$-catenin, and p120-catenin (p120) at the cell membrane in structures called adherens junctions (AJs) (1). During IDC tumor progression, AJs may be lost as a result of E-cadherin inactivation through epigenetic mechanisms that cause epithelial-to-mesenchymal transition (EMT), which is widely believed to mark the conversion to malignancy. In contrast, invasive lobular carcinoma (ILC) is characterized by loss or inactivation of E-cadherin at the initiating stages of tumor development, which suggests a difference in tumor etiology compared with IDC (2-4).

Catenins respond differentially to functional loss of E-cadherin. Unlike $\beta$-catenin, p120 is not degraded in human ILC, but instead accumulates in the cytoplasm and nucleus, where it has been proposed to regulate processes that influence invasiveness (5). Whereas cytosolic p120 coincides with loss of E-cadherin in ILC, IDC shows a 120 expression pattern that seems unrelated to E-cadherin and $\beta$-catenin status, including complete loss of p120 expression in approximately $10 \%$ of cases (6). These characteristics have advocated $\mathrm{p} 120$ expression and localization as a decisive marker in the differential diagnosis between IDC and ILC (7). Abnormalities in p120 expression have been reported in a variety of tumors, ranging from complete loss of protein expression to

Conflict of interest: The authors have declared that no conflict of interest exists. Citation for this article: J Clin Invest. 2011;121(8):3176-3188. doi:10.1172/JCI41695 accumulation (8). Thus, depending on tumor type, $\mathrm{p} 120$ may play a role as an oncogene or exert tumor suppressor functions.

Cytoplasmic overexpression of 120 results in a branching phenotype as a consequence of inhibition of the small GTPase RhoA, probably through indirect activation of the other Rho family members, Rac1 and Cdc42 (9). Here, p120 may function as a Rho-GDP dissociation inhibitor (RhoGDI) by directly binding RhoA, leading to inhibition of stress fiber-mediated contractility and thus increasing motility (10). Rho proteins regulate not only cell morphology, but also gene expression, cell proliferation, and survival (11). Key downstream effectors of Rho GTPase signaling are the Rho-associated kinases Rock1 and Rock2 (12), which regulate the actin cytoskeleton and cellular metabolism through phosphorylation of downstream effectors, such as cofilin (13) and myosin light chain (MLC) (14). In cancer, activation or overexpression of Rho GTPases and downstream Rock signaling components correlates with invasion, angiogenesis, and overall aggressiveness of tumors of the gastrointestinal tract, testis, and bladder (15-18).

Myosin phosphatase Rho-interacting protein (Mrip; also known as Mprip or $\mathrm{p} 116^{\mathrm{RIP}}$ ) is a ubiquitously expressed protein that was previously identified as a direct RhoA binding partner in mouse and human cells, resulting in inhibition of RhoA-mediated functions (19, 20). Mrip causes actin filament disassembly, probably by acting as a scaffold for multiple actin remodeling proteins, like RhoA and myosin phosphatase, and localizing them to actin filaments $(21,22)$.

Here, we have used well-defined mouse models of human ILC to show that loss of E-cadherin leads to translocation of p120 to the cytosol. In this setting, cytosolic p120 showed preferential binding to Mrip, an interaction that relieved the antagonistic effects of p120 and Mrip on the Rho/Rock signaling pathway. As a conse- 
A Adenocarcinoma
Wcre;Trp53"In!
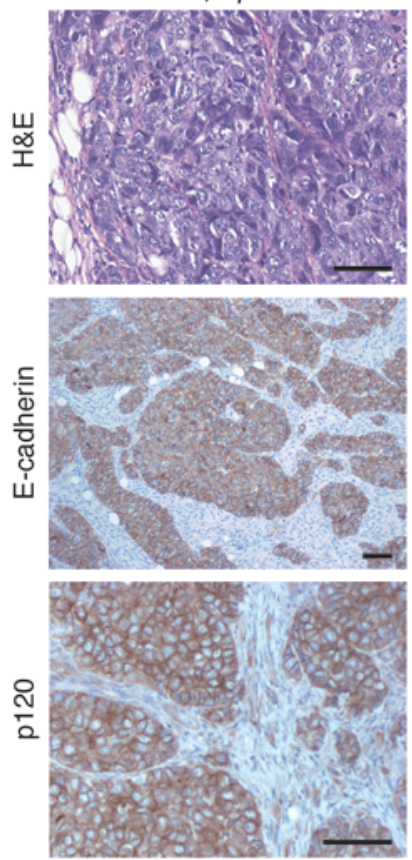

B

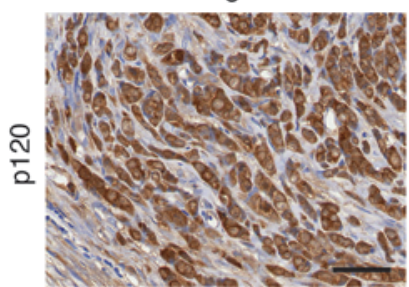

mILC

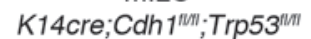
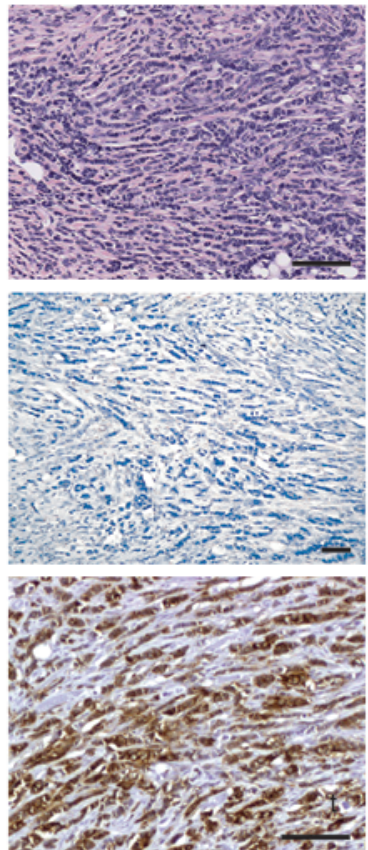

C

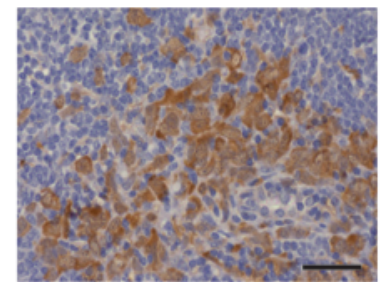

$\operatorname{Trp} 53^{\Delta \Delta-2}$

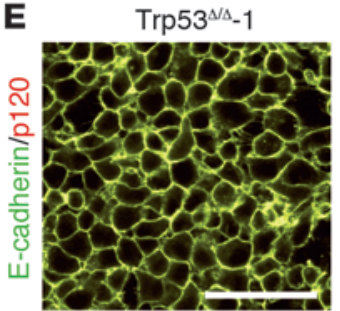

mILC-1

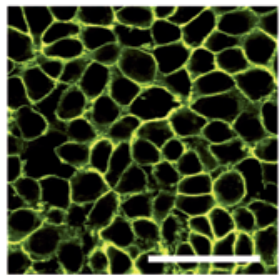

mILC Wcre;Cdh ${ }^{1 m n} ; \operatorname{Trp} 53^{m n n}$
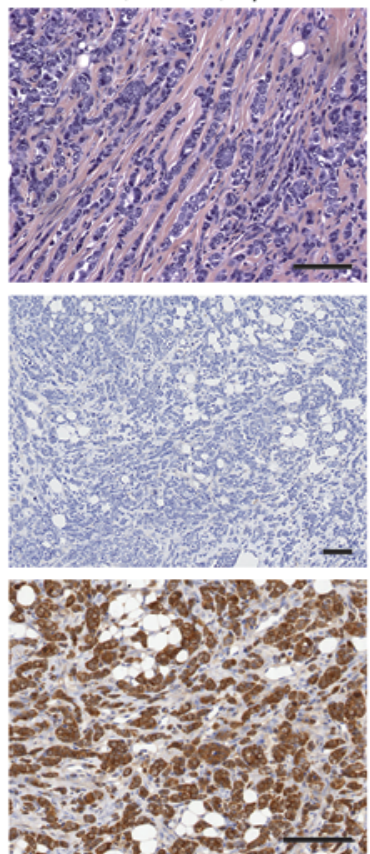

D
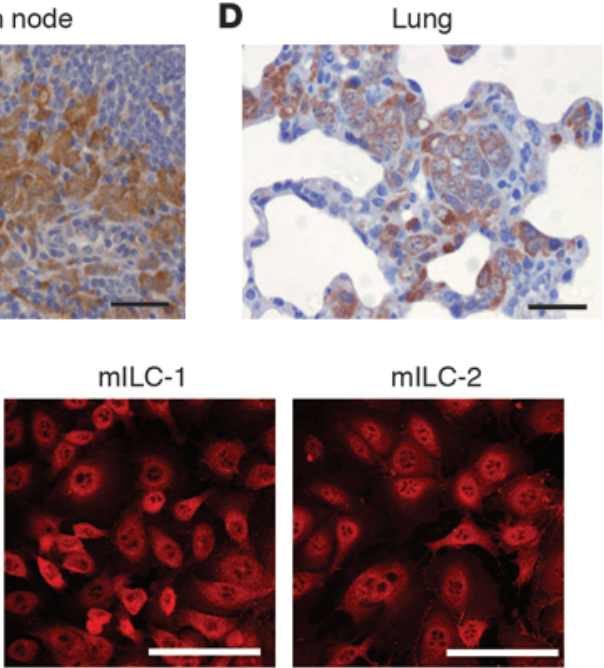

mILC-2

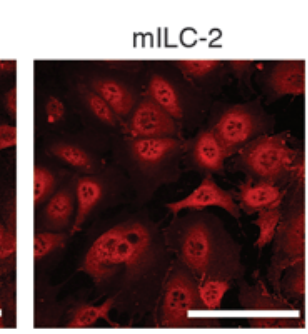

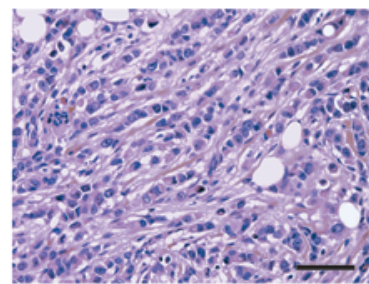
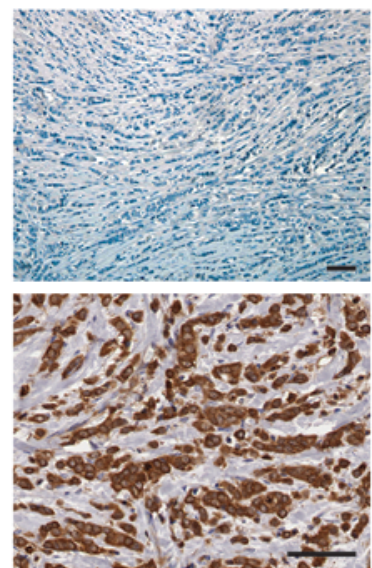

Figure 1

Loss of E-cadherin results in translocation of p120 to the cytosol. (A) Comparative histochemistry on mammary tumors derived from

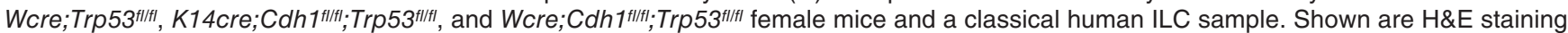
and immunohistochemistry for E-cadherin and p120. Note the striking resemblance of p120 localization in human ILC and mILC. (B-D) p120 translocation is retained during metastasis of mILC. (B) Invasive cells from a primary mILC. (C and D) Examples of distant metastatic mILC in a caudal lymph node (C) and lungs (D). All samples shown are from Wcre;Cdh1flffl;rp53 fl/fl female mice. (E) Cytosolic localization of p120 in mILC cell lines. Trp53 ${ }^{\Delta / \Delta}$ and mILC cell lines were stained for E-cadherin (green) and p120 (red). Scale bars: $30 \mu \mathrm{m}$.

quence, RhoA and downstream Rock1 signaling was active, resulting in anoikis resistance and in vivo tumor growth of metastatic lobular breast cancer.

\section{Results}

Somatic loss of E-cadherin leads to translocation of p120 to the cytosol. We previously showed in a conditional mouse model of human ILC that loss of E-cadherin is causally related to acquisition of anoikis resistance and subsequent metastasis of mammary tumor cells (4). Using mouse ILC (mILC) as a model system, we set out to investigate the consequences of somatic loss of E-cadherin for AJ complex members. First, we analyzed the expression and localization of $\beta$-catenin and activity of canonical Wnt signaling. In agreement with previous studies $(23,24)$, somatic inactivation of E-cadherin did not induce accumulation of $\beta$-catenin and subsequent activation of canonical Wnt signaling in mILC (Supplemental Fig- 

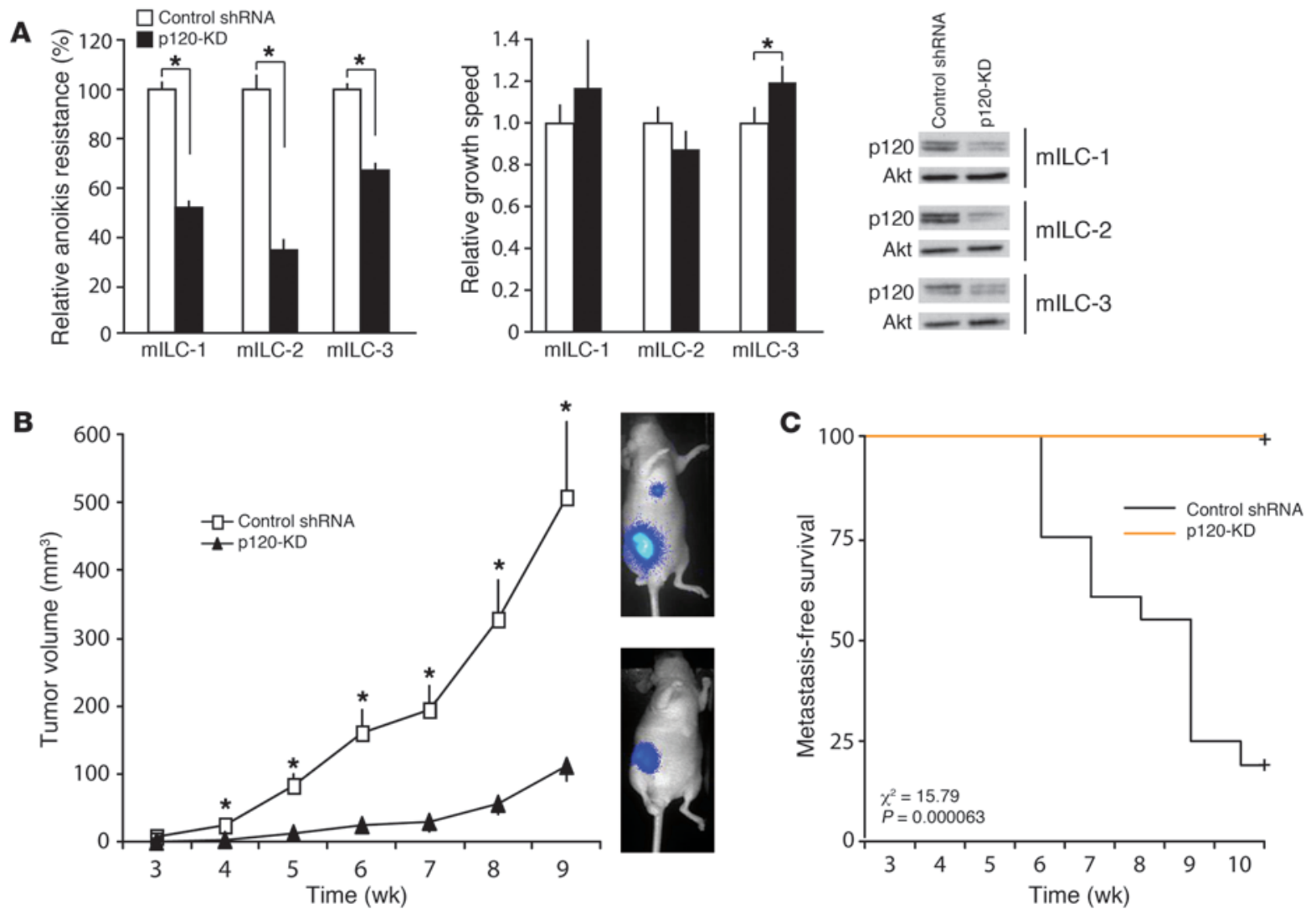

Figure 2

p120 controls mILC anoikis resistance and tumor growth. (A) Cells were transduced with viruses carrying mouse p120 shRNAs and grown for 4 days in the absence and presence of anchorage, after which anoikis resistance $(n=4)$ and growth speed $(n=6)$, respectively, were analyzed. Western blotting for p120 is shown to depict the extent of shRNA KD in 3 independent mILC cell lines. Akt was used as a loading control. Error bars represent SD of triplicate measurements. ${ }^{\star} P<0.001$. (B) Luciferase-expressing mILC control shRNA or p120-KD cells were orthotopically transplanted into recipient mice, and tumor volume was measured longitudinally. Error bars represent SEM. ${ }^{\star} P<0.05$ vs. p120-KD. $n=11$ (p120-KD); 16 (control shRNA; of which 12 survived at 7 wk, 10 at 8 wk, 9 at 9 wk). (C) Kaplan-Meier metastasis-free survival plot of the mice in B ( $n=11$ [p120-KD]; 16 [control shRNA]). Recipient animals were followed in time and sacrificed when bioluminescence was detected in the lungs (>500 counts).

ure 1; supplemental material available online with this article; doi:10.1172/JCI41695DS1).

Because cytoplasmic p120 expression can be used as a decisive tool in the differential diagnosis between IDC and ILC and has previously been shown to correlate with invasiveness and metastatic capacity $(5,7,22)$, we closely examined p120 expression in mILC. Using immunohistochemistry, we observed that p120 expression was indeed retained in primary mammary tumors from

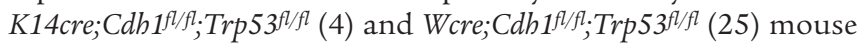
models. Whereas E-cadherin-expressing benign adenocarcinomas showed distinct membrane-localized p120, mILC samples exclusively displayed cytosolic p120, identical to their human analog (Figure 1A). Immunohistochemistry also revealed that cytosolic expression of p120 was retained in mILC cells that had invaded surrounding mammary tissue and in metastatic cells that had colonized a caudal lymph node and the lungs (Figure 1, B-D). In mILC cell lines $(n=8)$, p120 also localized to the cytosol (Figure $1 \mathrm{E})$. Loss of E-cadherin did not result in accumulation of $\mathrm{p} 120$, as determined by Western blotting and expression profiling of primary tumors and their cell line derivatives (data not shown). Based on molecular weight, we detected concomitant expression of p120 isoforms 1 and 3 in the E-cadherin-proficient cell line $\operatorname{Trp} 53^{\Delta / \Delta}$ and in mILC cell lines (data not shown and Figure 2A).

p120 regulates anoikis resistance of $m I L C$ cells. Loss of a functional AJ is a key event that leads to anchorage independence of mILC cells (4). As p120 is translocated to the cytosol in mILC, we wondered whether it contributes to the regulation of anoikis resistance. To test this, we cloned a previously published siRNA against mouse p120 (26) into a lentiviral system and established knockdown (KD) of both p120 isoforms upon transduction, which did not inhibit anchorage-dependent proliferation or viability of these cell lines (Figure 2A). By growing cells in suspension and assaying apoptosis, we found a near-perfect correlation between anoikis resistance and in vivo metastasis (4), a parallel we did not observe using growth in soft agar (data not shown). Interestingly, anoikis resistance was significantly inhibited upon p120 KD in 3 independent mILC cell lines (Figure 2A), which suggests that p120 facilitates anchorage-independent survival. A control shRNA vector targeting human p120 (containing 3 mismatches compared with the mouse sequence) affected neither anoikis resistance nor anchorage-dependent growth. To determine whether p120-controlled anoikis resistance also contributes to tumor growth in vivo, we 
A
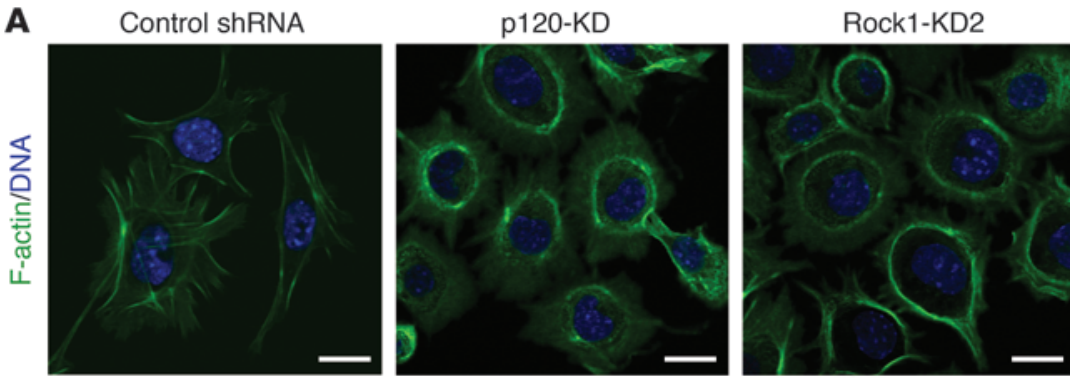

B

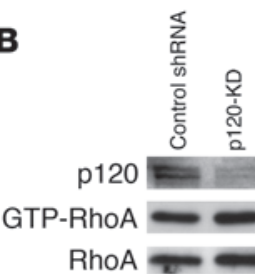

c

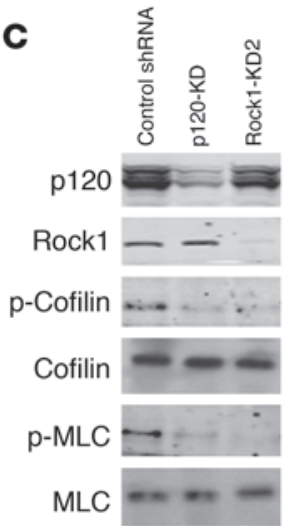

\section{Figure 3}

p120 controls activation of Rock signaling in mILC. (A) Control shRNA and p120-KD mlLC-1 cells were stained for F-actin using Alexa Fluor 488-conjugated phalloidin. Rock1-KD2 mILC-1 cells were used as a positive control for actin depolymerization. DNA was visualized using DAPI. Scale bars: $5 \mu \mathrm{m}$. (B) GST-Rhotekin pulldown assays were performed on lysates from control shRNA or p120-KD cells and blotted for GTP-bound RhoA and total RhoA. Shown is 1 representative experiment of 3 . (C) Western blot analysis showing the inhibitory effect of p120 KD on phosphorylation of cofilin and MLC. Total cofilin and MLC are shown as loading controls. Rock1-KD2 mILC -1 cells were used as a positive control for inhibition of cofilin and MLC phosphorylation. performed orthotopic transplantations of luciferase-expressing mILC- 1 cells in which p120 was genetically silenced. p120 KD in mILC-1 cells resulted in a robust inhibition of tumor growth (Figure 2B), which indicates that inhibition of anoikis resistance prevented colonization and subsequent outgrowth. Also, p120 KD prevented lung metastasis, whereas control shRNA mILC-1 cells readily metastasized (Figure $2 \mathrm{C}$ ). These findings indicate that $\mathrm{p} 120$ controls the regulation of mILC tumor growth through acquisition of anoikis resistance.

p120 controls activation of Rho/Rock signaling in mILC. Overexpression of 120 has been shown to inhibit the activation of RhoA, possibly by functioning as a RhoGDI or, alternatively, through Vav-2-mediated activation of $\operatorname{Rac} 1(9,10)$. To analyze whether cytosolic p120 regulates RhoA in our system, we assessed actin polymerization, an indirect but functional readout of active Rho/Rock signaling, in 3 independent mILC cell lines. Surprisingly, we detected abundant stress fibers using phalloidin staining, which was substantiated by GTP pulldown assays and Western blotting for RhoA (Supplemental Figure 2, A and B). Furthermore, we observed phosphorylation of cofilin, which was specifically inhibited upon administration of the Rock inhibitors Y-27632 or hydroxyfasudil (OH-fasudil) (Supplemental Figure 2C). We therefore conclude that Rho/Rock signaling is active in the presence of endogenous cytosolic p120 in mILC.

Given the fact that RhoGDI function has been attributed to p120, we next determined the effect of p120 overexpression on our cells. Only upon induction of high levels of p120 overexpression, mILC cells showed a branching phenotype (Supplemental Figure 3), which is strongly linked to a p120-mediated inactivation of RhoA (9). We therefore conclude that mILC cells - depending on the level of exogenous overexpression - conform to the notion that cytosolic p120 may inhibit RhoA.

Next, we set out to determine whether p120 directly affects RhoA and downstream signaling via Rock. We performed a stable
KD of $\mathrm{p} 120$ and tested the effect on actin polymerization. Filamenous actin (F-actin) was clearly reorganized and showed a marked reduction in stress fiber formation identical to that in Rock1-KD mILC-1 cells, used as a control (Figure 3A). These results imply that $\mathrm{p} 120$ regulates the actin cytoskeleton, possibly through modulation of Rho and Rock. Despite the effect on actin remodeling, we did not observe an effect of p120 KD on GTP-bound RhoA (Figure 3B), which indicates that in mILC, p120 does not directly regulate the activity of RhoA. Next, we analyzed Rho/Rock signaling by probing the phosphorylation status of the 2 prominent downstream Rock targets, cofilin and MLC, upon inhibition of p120. In line with the observed effects on actin depolymerization, we observed a reduction in phosphorylated cofilin and MLC similar to the inhibition in Rock1-KD mILC-1 cells (Figure 3C), which suggests that $\mathrm{p} 120$ may regulate anoikis resistance through targets downstream of Rho and Rock. To substantiate the notion that p120 exerts its effect as a result of its cytosolic localization, we restored p120 to the cell membrane by lentiviral reconstitution of E-cadherin. As expected, relocalization of endogenous p120 resulted in a reduction of phosphorylated MLC (Supplemental Figure 4, A-D). To uncouple E-cadherin-controlled cell-cell adhesion from the p120-mediated and localization-dependent regulation of Rock signaling, we cloned a lentiviral version of a fusion construct in which the extracellular domain of E-cadherin was replaced by that of the IL-2 receptor (IL-2R) (27), and transduced mILC-1 cells. Indeed, upon expression of the IL2R-Ecadcyto fusion protein, we observed inhibition of Rock signaling independent of homotypic AJ formation (Supplemental Figure 4D). Moreover, these results were functionally substantiated by a decrease in anoikis resistance upon relocalization of p120, using both wild-type E-cadherin and the IL2R-Ecad-cyto fusion protein (Supplemental Figure 4E). We conclude that localization of p120 regulates Rock-mediated anoikis resistance in mILC. 

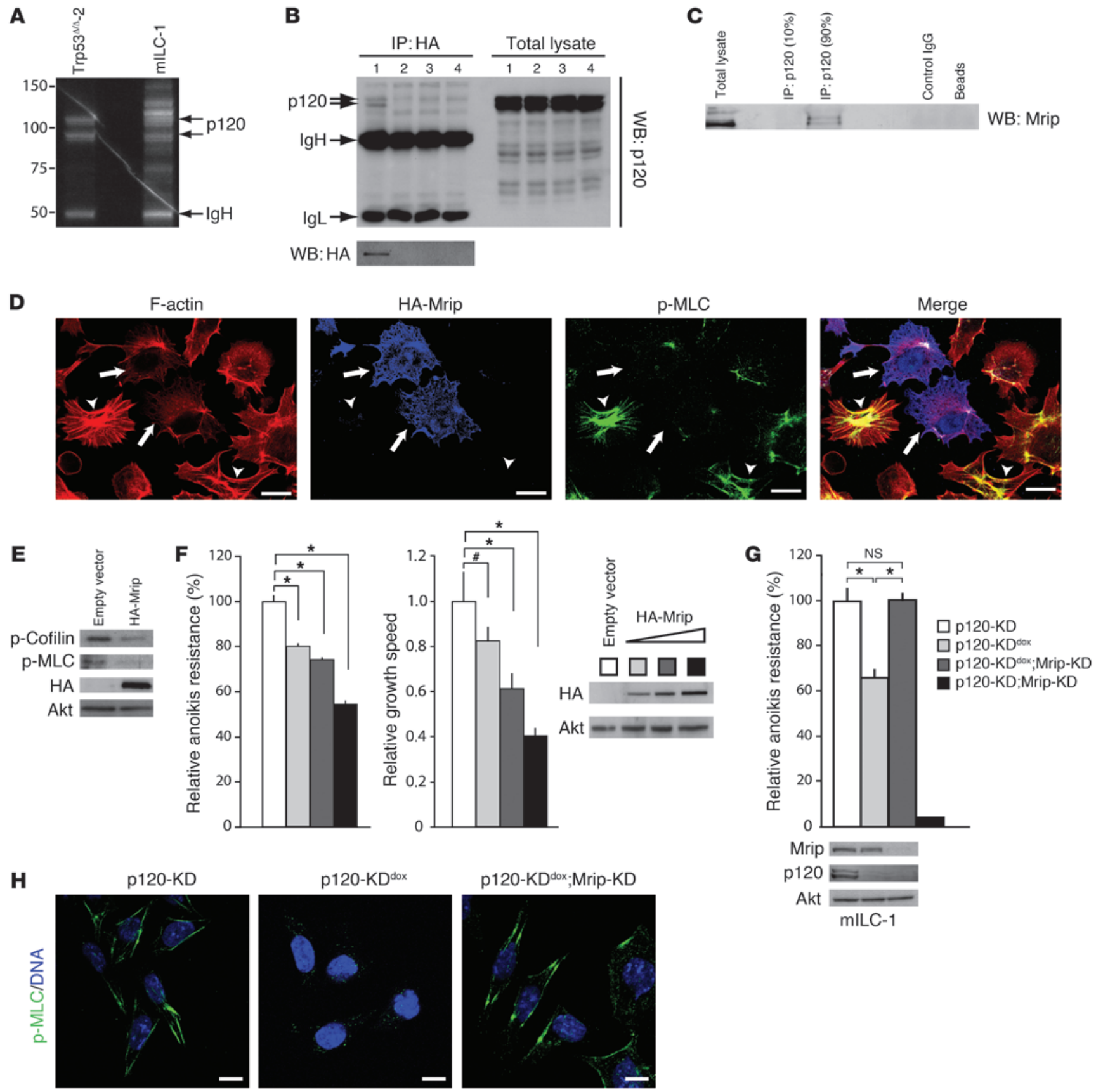

Figure 4

Interaction between p120 and Mrip facilitates Rock-dependent anoikis resistance. (A) IP of p120 from Trp53 $3^{\Delta / \Delta-2}$ and mILC-1 cells; differential bands were analyzed using MS. IgH, Ig heavy chain. (B) mILC-1 cells were transfected with HA-Mrip, subjected to co-IP using anti-HA antibodies, and blotted for p120. Lane 1, HA-Mrip; lane 2, empty vector; lane 3, no vector; lane 4, no transfection. Total cell lysates were blotted for p120 as input control. HA-Mrip expression is shown below. IgL, Ig light chain. (C) IP of endogenous p120 from mILC-1 cells, Western blotted and stained for Mrip. Also shown are IgG and beads-only controls. (D) HA-Mrip-transduced mILC-1 cells were mixed with empty vector-transduced cells and stained for F-actin (red), HA (blue), and phospo-MLC (green). Arrows, transduced cells; arrowheads, control cells. Scale bars: 5 um. (E) HA-Mrip-transduced cells were assayed for phospho-cofilin, phospho-MLC, and Akt (loading control). (F) mILC-1 cells were transduced with HA-Mrip using increasing viral titers, then assayed for anoikis resistance $(n=3)$ and growth speed $(n=6)$. Western blotting shows expression levels of HA-Mrip and Akt (loading control). ${ }^{*} P<0.001$; $P<0.005$. (G) mILC-1 cells containing dox-inducible shRNA sequences targeting 120 and constitutive Mrip shRNAs were assayed for anoikis resistance $(n=3)$. For the p120-KD;Mrip-KD group, no protein samples were harvested as a result of severe cell death. ${ }^{*} P<0.001$. (H) Activity of Rock signaling was assessed by staining for phospho-MLC. Scale bars: $5 \mu \mathrm{m}$. 

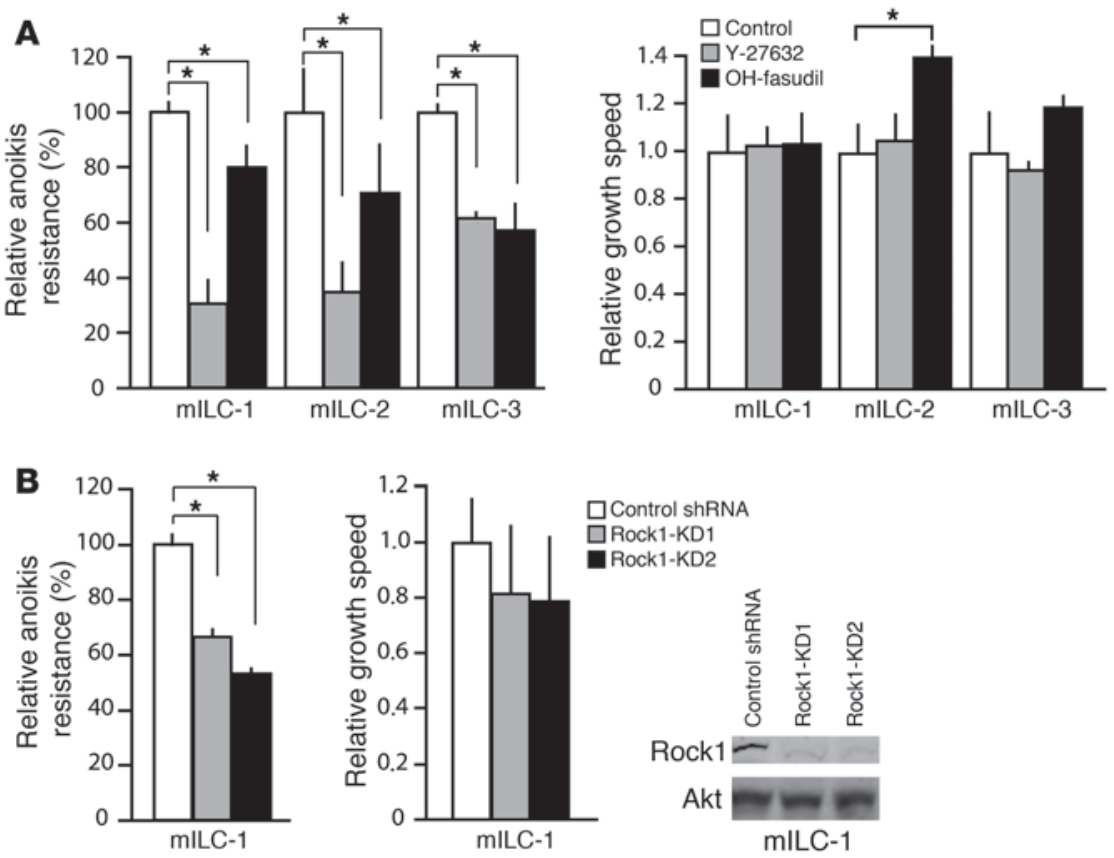

$\square$ Control ShRNA

\section{Rock1-KD1}

Rock1-KD2
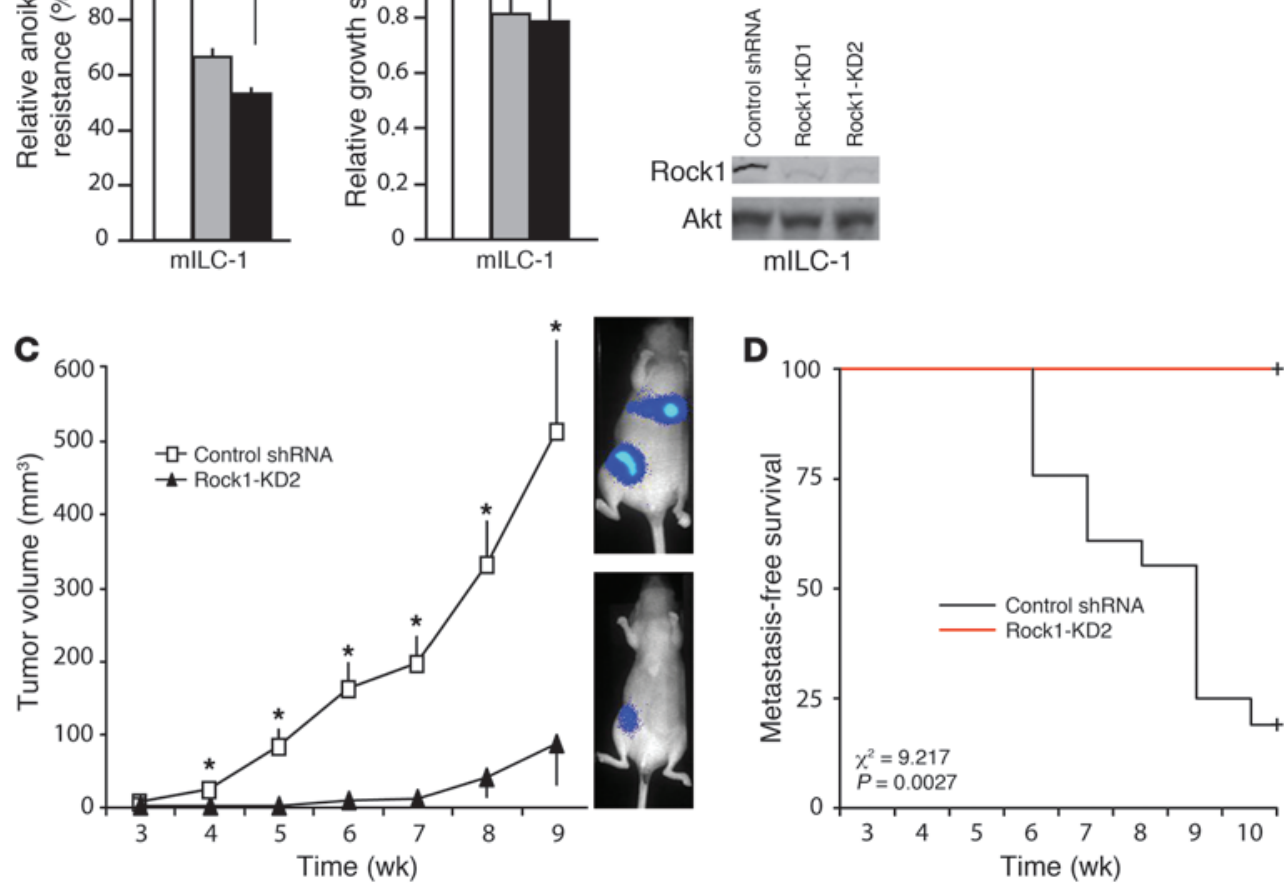

Figure 5

Rock signaling regulates mILC anoikis resistance and tumor growth. (A and $\mathbf{B}$ ) Cells were treated with Y-27632 or OH-fasudil (A) or transduced with viruses carrying shRNA sequences against mouse Rock1 (B) and grown for 4 days in the absence or presence of anchorage, after which anoikis resistance $(n=3)$ and growth speed $(n=6)$, respectively, were analyzed. Western blotting for Rock1 depicts the extent of KD of 2 independent Rock-1 shRNA sequences. Akt was used as a loading control. Error bars represent SD of triplicate measurements. ${ }^{*} P<0.05$. (C) Luciferase-expressing mILC-1 control shRNA or Rock1-KD2 cells were orthotopically transplanted into recipient mice, and tumor volume was measured longitudinally. Error bars represent SEM. ${ }^{*} P<0.05$ vs. Rock1-KD2. $n=6$ (Rock1-KD2); 16 (control shRNA; see Figure 2B). (D) Kaplan-Meier metastasis-free survival plot of the mice in C ( $n=6$ [Rock1-KD2]; 16 [control shRNA]). Recipient animals were followed in time and sacrificed when bioluminescence was detected in the lungs (>500 counts). Controls in $\mathbf{C}$ and $\mathbf{D}$ are duplicated from Figure 2, B and C, for purposes of comparison.

tion, as a prominent hit (mascot score, 1,702). We substantiated the 120 -Mrip interaction upon exogenous expression of HAtagged Mrip into mILC-1 cells and subsequent co-IP using antibodies against HA (Figure 4B). Moreover, we confirmed our MS data by demonstrating the interaction of the endogenous proteins by co-IP and subsequent Western blot analysis (Figure 4C). These biochemical data demonstrate that $\mathrm{p} 120$ interacts with Mrip in mILC.

Since Mrip exerted inhibitory effects on Rho function, and p120 acted as a modulator of Rho/Rock signals, we hypothesized that the interaction between cytosolic p120 and Mrip impedes their inhibitory effects downstream of Rho. If so, overexpression of Mrip should lead to inhibition of Rock signaling in mILC cells. Indeed, lentiviral-mediated overexpression of HA-tagged Mrip in mILC-1 cells resulted in a decrease in phosphorylation levels of MLC and a reduction in stress fibers (Figure 4D). Subsequent Western blotting confirmed these findings, showing a marked reduction in phosphorylated levels of cofilin and MLC (Figure $4 \mathrm{E}$ ). To test the functional consequences of these findings, we transduced mILC-1 cells with HA-tagged Mrip using an increasing multiplicity of infection (Figure 4F). Upon transfer to anchorage independence, we indeed observed that anoikis resistance of mILC cells gradually decreased with increasing protein levels of Mrip (Figure 4F). Overexpression of Mrip also reduced anchorage-dependent growth (Figure 4F), which indicates that proximal interference of actin polymerization is not

Cytoplasmic 120 controls Rho/Rock signaling through binding of Mrip. In light of the fact that 120 has been described as a RhoGDI (9), we set out to identify possible intermediates that would explain active Rho/Rock signaling in the presence of cytosolic p120. To this end, we performed IP on endogenous p120 from mILC- 1 and $\operatorname{Trp} 53^{\Delta / \Delta}-2$ cells and performed mass spectrometry (MS) on the differential p120 binding partners in mILC cells (Figure 4A). From the resulting data, we identified Mrip, an antagonist of Rho func- tolerated in the presence of anchorage. Next, we tested whether inhibition of Mrip leads to decreased Rock signaling, since both p120 and Mrip antagonized the Rho/Rock axis. Using stable shRNA-mediated KD of Mrip, we observed that Mrip inhibition was not tolerated and resulted in cell death, independent of cellular context (Figure 4G and data not shown). Since inhibition of p120 or overexpression of Mrip resulted in decreased Rock signaling and subsequent anoikis resistance, we reasoned that simul- 
A
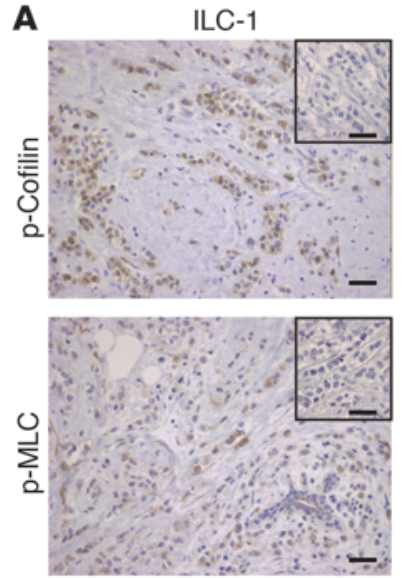

ILC-2
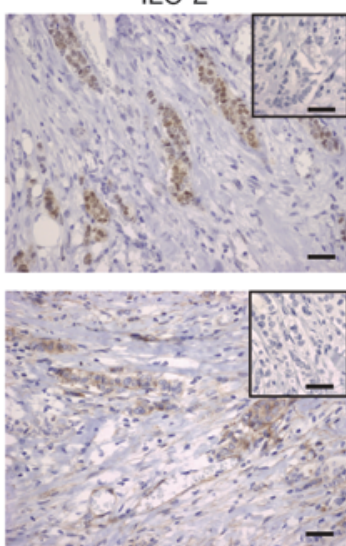

\section{B}

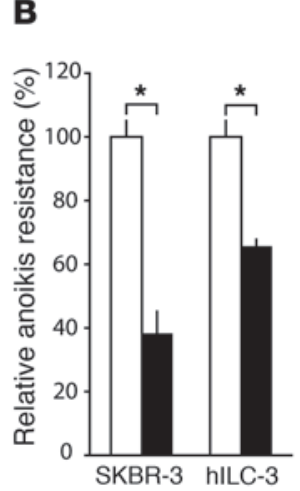

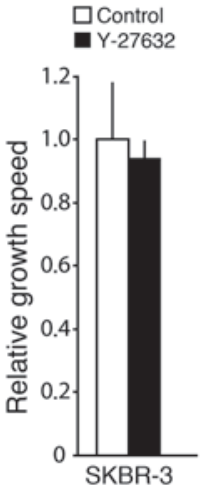

C SKBR-3 p120-KD

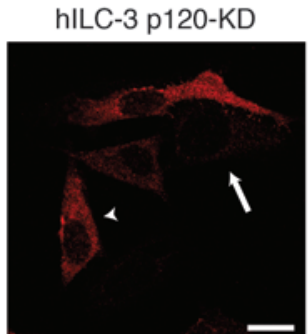

\section{D}
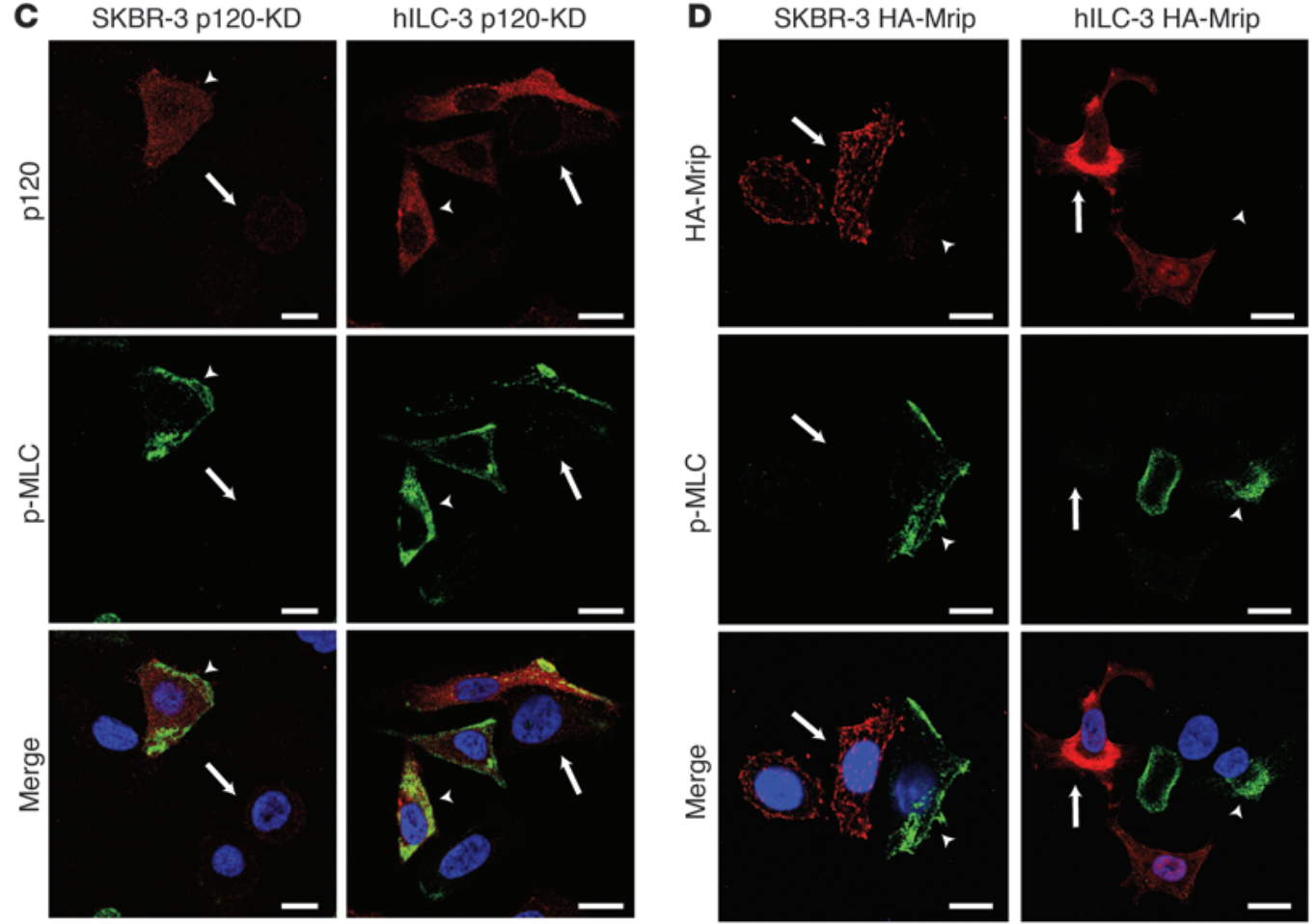

\section{Figure 6}

Active Rock signaling contributes to growth and survival of human ILC. (A) Human ILC samples were stained for phosphorylated cofilin and phosphorylated MLC by immunohistochemistry. Insets show the effect of $\lambda$-phosphatase treatment, indicating specificity of the antibodies. Scale bars: $30 \mu \mathrm{m}$. (B) SKBR-3 and primary hILC-3 cells were cultured in the absence of anchorage and treated with $10 \mu \mathrm{M}$ Y-27632. After 4 days, anoikis resistance $(n=3)$ and growth speed $(n=6)$ were determined. Error bars represent SD of triplicate measurements. ${ }^{*} P<0.005$. (C) SKBR-3 and primary hILC-3 cells were transduced with viruses carrying shRNA sequences against human p120. Cells were stained for DNA using DAPI (blue), p120 (red), and p-MLC (green). Arrowheads, nontransduced cells; arrows, transduced p120-KD cells. Note the reduction in phosphorylated MLC upon p120 KD. Scale bars: $5 \mu \mathrm{m}$. (D) SKBR-3 and hILC-3 cells were transduced with viruses carrying HA-tagged Mrip. Cells were stained for DNA using DAPI (blue), HA (red), and p-MLC (green). Arrows, HA-Mrip-expressing cells; arrowheads, nontransduced cells. Scale bars: $5 \mu \mathrm{m}$.

taneous inhibition of $\mathrm{p} 120$ and Mrip should restore active Rock signaling and reestablish anoikis resistance. To investigate this, we generated mILC- 1 cells carrying a doxycycline-inducible (doxinducible) p120 shRNA construct (p120-KDdox) and transduced these cells with a constitutive Mrip shRNA-expressing construct (p120-KD dox;Mrip-KD). As expected, culturing p120-KD ${ }^{\text {dox; }}$;rip$\mathrm{KD}$ cells in the absence of dox resulted in massive cell death (Fig- ure 4G). However, dox-inducible inhibition of p120 fully rescued anoikis resistance in the context of Mrip KD. In agreement with these findings, we observed that simultaneous KD of p120 and Mrip restored Rock signaling activity (Figure $4 \mathrm{H}$ ). These data indicate that Rho/Rock signaling is active as a result of the p120-Mrip interaction, thereby promoting anoikis resistance. Moreover, they imply specificity for the p120 and Mrip siRNA sequences, since 
inducible inhibition of p120 resulted in a full reversal of the anoikis phenotype upon Mrip KD.

Rock controls anoikis resistance and in vivo tumor growth of mILC. As Rho and Rock are refractory to pharmacological inhibition, we set out to test whether inhibition of this pathway influences anoikis resistance of mILC. Inhibition of Rho using C3 transferase or Rock using Y-27632 or OH-fasudil reduced anoikis resistance of mILC cells, whereas identical concentrations had no inhibitory effects on anchorage-dependent growth (Supplemental Figure 2D and Figure $5 \mathrm{~A}$ ). To extend these findings, we tested whether genetic inhibition of Rock 1 would result in inhibition of anoikis resistance. Indeed, using 2 independent shRNAs, we observed a strong reduction of anoikis resistance, whereas anchorage-dependent growth was not significantly affected (Figure 5B). To validate these observations in vivo, we orthotopically transplanted Rock1-KD mILC-1 cells in recipient female animals and followed tumor growth and dissemination longitudinally. Similar to the effects seen upon transplantation of $\mathrm{p} 120-\mathrm{KD}$ cells, we observed a retardation of tumor growth compared with control animals (Figure 5C). Moreover, Rock-mediated inhibition of tumor growth also prevented mILC lung metastasis (Figure 5D). Together, these data illustrate the importance of Rho/Rock signaling in the regulation of mILC anoikis resistance and in vivo tumor growth.

Rock signaling controls anoikis resistance in buman ILC. Because findings from our mouse models of human ILC showed an important role for Rock signaling in context-dependent tumor growth, we wondered whether this also holds true for human disease. We began to investigate this using immunohistochemistry on primary ILC tumor tissues. As in mILC, we detected mutual expression of phosphorylated cofilin and phosphorylated MLC in the majority of human ILC samples (13 of 17 and 11 of 17, respectively; Figure $6 \mathrm{~A})$. When we compared the extent of phosphorylated cofilin in IDC versus ILC, we found a significant increase, from $46 \%$ to $77 \%$ ( $n=91$ [IDC], 17 [ILC]; $P<0.01$, Pearson exact $\chi^{2} 2$-sided), which strengthens the notion that activation of Rock may be a feature of ILC. To address whether Rock activation has functional implications for ILC survival, we tested the effect of Rock inhibition on anoikis resistance of an E-cadherin mutant, luminal-type cell line (SKBR-3), and on primary human ILC cells derived from pleural effusion fluids (hILC-3). As in our mouse model, Y-27632 induced a significant decrease in anoikis resistance of SKBR-3 and hILC-3 cells (Figure 6B). Anchorage-dependent growth of SKBR-3 was not affected by Y-27632, in agreement with data obtained in our mILC lines. In concordance with our findings in MILC, we also observed an inhibition of anoikis resistance when SKBR-3 was treated with $\mathrm{C} 3$ and OH-fasudil (Supplemental Figure 5). Because hILC-3 did not proliferate under adherent culture conditions (data not shown), we could not assess anchorage-dependent growth. To confirm that p120 regulates Rock signaling analogous to our mouse model, we transduced SKBR-3 and hILC-3 cells with lentiviruses expressing P120 shRNA and probed for phosphorylated MLC. Indeed, p120 KD inhibited MLC phosphorylation (Figure 6C). Moreover, transduction of HA-tagged Mrip resulted in reduced levels of phosphorylated MLC (Figure 6D), which suggests that human ILC also functionally depends on the p120-Mrip interaction for activation of Rock signals. Our findings confirmed the dependence of human ILC on active Rock signals for the regulation of anchorage independence and corroborated our finding that the p120-Mrip interaction facilitated active Rock signaling, which was required for anchorage-independent survival.

\section{Discussion}

Clinically, p120 localization can be used as a determinant in the differential diagnosis between ductal and lobular tumor types (7). Whereas cytosolic p120 coincides with E-cadherin loss in ILC, IDC shows an expression pattern that seems unrelated to E-cadherin and $\beta$-catenin status, including complete loss of p120 expression in approximately $10 \%$ of the cases (6). Since early loss of E-cadherin is causal for ILC formation, we believe this evokes biochemical signals that differ from inactivation of E-cadherin during later stages.

Our data demonstrate that cytosolic p120 exerted oncogenic properties that became eminent in the absence of anchorage. Upon loss of E-cadherin, p120 translocated to the cytosol, where it bound the inhibitor of Rho function Mrip. We believe this insight on how p120 may facilitate anoikis resistance through indirect activation of the Rho/Rock signaling pathway to be novel. Several lines of evidence concur with the notion that p120 plays an important role in regulating anchorage independence of cancer cells. For instance, it was recently shown that p120 controls growth in soft agar of E-cadherin-expressing MDCK cells, which rely on overexpression of exogenous dominant active oncogenes such as Src or Rac for anchorage independence. Interestingly, p120 KD in Srcor Rac-transformed MDCK cells inhibited anchorage-independent growth in soft agar, an effect that could be rescued by Rock inhibition using Y-27632 (28). Furthermore, recent data showed that in a human IDC cell line in which E-cadherin is silenced by methylation, MDA-MB-231 (MM231) cells, p120 also controls anchorage-independence - although in these cells, it is controlled by Rac1-dependent activation of the MAPK pathway. Here, it was hypothesized that E-cadherin loss during tumor progression may relieve the negative regulation of Ras through p120-dependent activation of Rac in a cell type-specific manner (29). Since it is assumed that active Rac counterbalances the activity of Rho (30), these findings suggest that, under these settings and in these cell types, active Rho/Rock signaling does not induce anchorage independence. As expected, when cultured in the absence of anchorage and treated with Y-27632, MM231 cells did not show decreased anoikis resistance (data not shown). Moreover, Rac signaling did not appear to be the driver of anoikis resistance of mILC, because dominant-negative RacN17 and the Rac inhibitor NSC-23766 did not influence anchorage-independent survival of mILC (data not shown). Moreover, our MS analysis gave no indication that p120 interacts with cadherin-11 in mILC, which clearly indicates the differences in model systems studied. We refrained from using soft agar as a substrate for the anoikis experiments because it does not provide predictive value for in vivo metastasis when using our mouse cell lines. An explanation for this could reside in the fact that soft agar often contains denatured collagen, which is known to trigger binding of $\alpha 5 \beta 1$ and $\alpha \mathrm{v}$ integrins and subsequent activation of Rac through exposed RGD binding (31-33). It is still unclear how Rho is activated in ILC. Given our finding that inhibition of p120 and Rock led to context-dependent effects on tumor cell survival, we believe that upon transition to anchorage independence, cells undergo morphological changes that may activate Rho and its downstream targets (34). Alternatively, the activation of Rho in ILC may also be triggered by (autocrine) activation of receptor-mediated signaling (35-37). We anticipate that the level and mode of activation may be different in the context of Rho-mediated induction of apoptosis, which can be triggered by strong exogenous cues, such as LPA and ethanol $(38,39)$. Interestingly, Rock signaling can also be activated upon induction of apoptosis. In this 
A E-cadherin ${ }^{+/+}\left(\operatorname{Trp}^{2} 3^{\Delta / \Delta}\right)$

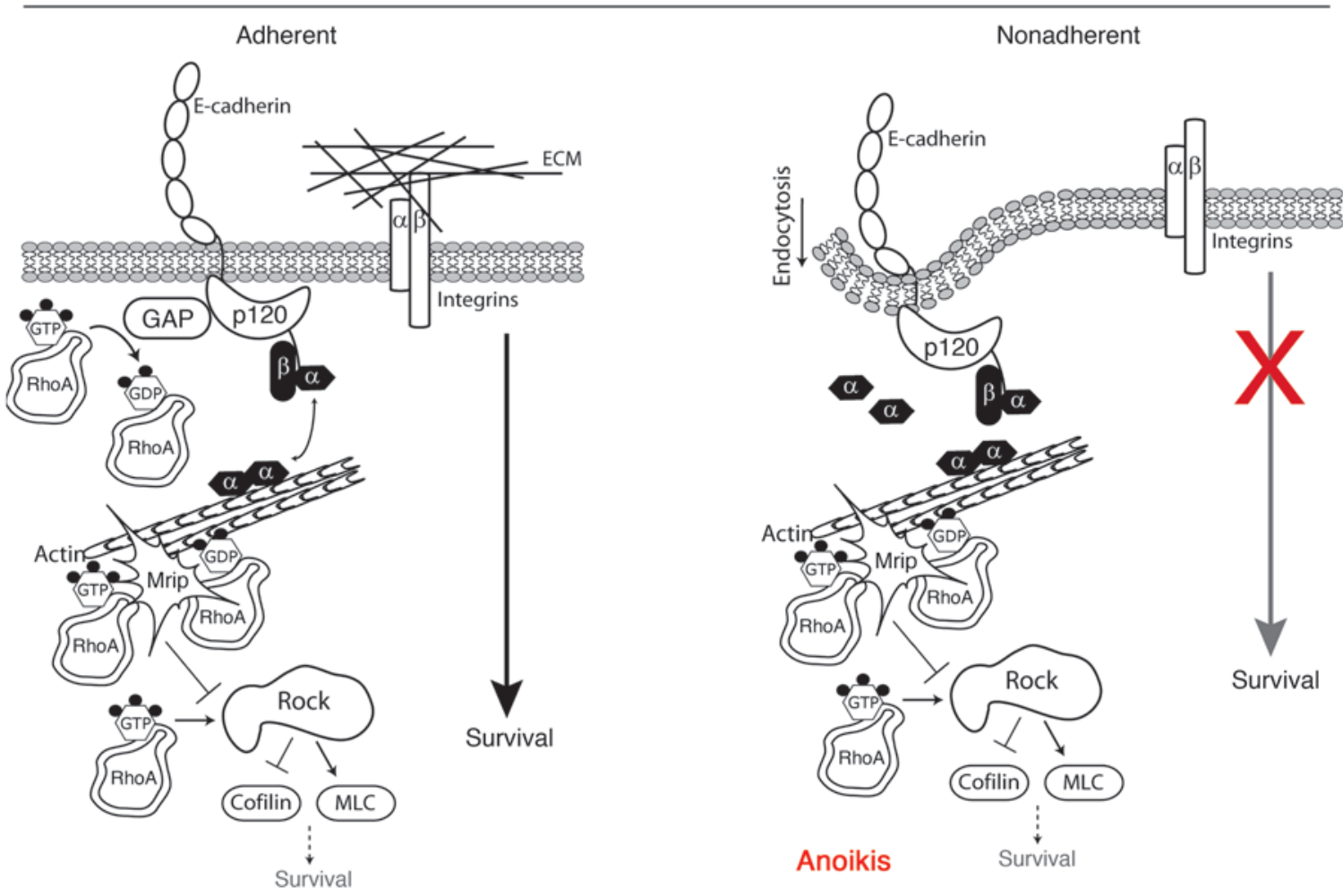

B E-cadherin ${ }^{\Delta \Delta}$ mouse ILC $\left(\operatorname{Trp}^{\Delta} 3^{\Delta \Delta}\right)$ and human ILC

Adherent

Nonadherent
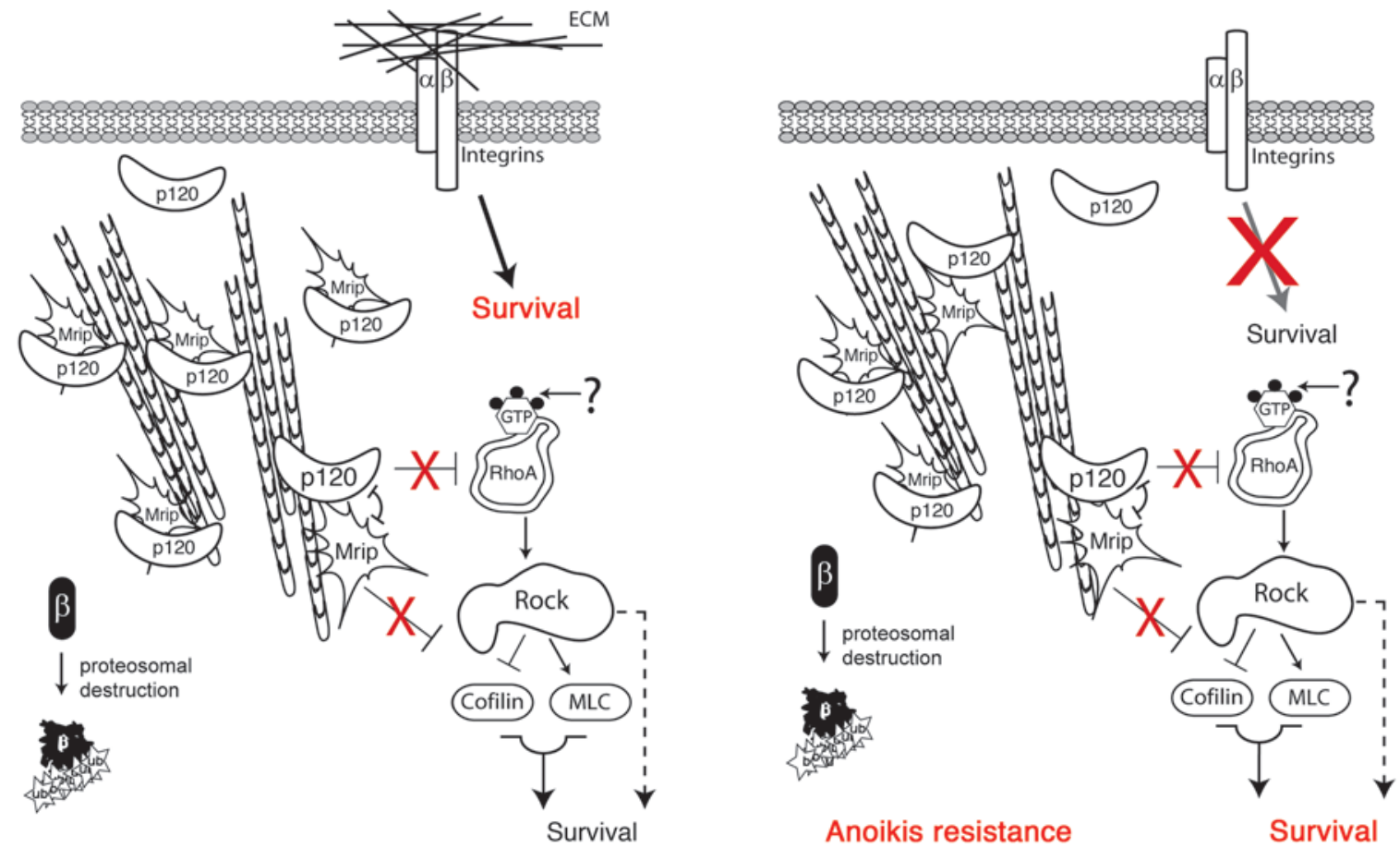


\section{Figure 7}

Model for p120-mediated regulation of anoikis resistance in ILC upon loss of E-cadherin. (A) Under benign conditions and in the presence of a functional AJ, Rho levels and cellular survival mechanisms are balanced through a fine-tuned equilibrium of protein interactions and signaling pathways (left). When cells are deprived of ECM-integrin interactions, a potent outside-in survival signal is impeded, resulting in anoikis because of a lack of compensatory survival mechanisms (right). (B) Upon early mutational inactivation of E-cadherin in human ILC and

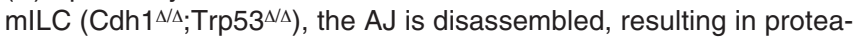
somal degradation of $\beta$-catenin. More importantly, p120 translocates to the cytosol, where it binds Mrip, an antagonist of Rho/Rock function. Since p120 has RhoGDI functions, this interaction will alleviate the inhibitory functions of both $\mathrm{p} 120$ and Mrip, leading to active Rho/Rock signaling by an unknown mechanism. Although activation of Rho and Rock may have a positive effect on cellular survival in the presence of ECM, these signals will be overridden by powerful integrin-mediated survival cues (left). However, when ILC cells transfer to anchorage independence, the survival signals that emanate from Rho and Rock will rescue the cells, leading to anoikis resistance of ILC (right).

scenario, activation of caspases promote Rho-independent cleavage and constitutive activation of Rock1, leading to membrane blebbing and disruption of nuclear integrity (40-42). In contrast, human ILC and mILC $\left(\mathrm{Cdh} 1^{\Delta / \Delta} ; \operatorname{Tr} p 53^{\Delta / \Delta}\right)$ controlled anoikis resistance by continuous activation of Rho-dependent Rock signaling (Supplemental Figure 2). We therefore think that the difference in functional outcome as a result of actin-myosin contractility during apoptosis may be caused by differences in Rock 1 activation levels, or that activation of Rock1 by apoptosis-inducing cues (such as FasL or a combination of TNF- $\alpha$ and cycloheximide) may confer modulation of additional pathways that facilitate membrane blebbing and relocalization of fragmented DNA into apoptotic bodies. Finally, although our data showed that cytosolic p120 regulated Rock signals and subsequent anoikis resistance in mILC, we cannot exclude the possibility that loss of p53 affects Rock signaling when concomitantly inactivated with E-cadherin. Regardless of the exact mechanism, it is clear that signaling events downstream of p120 show differences based on context and cell type.

In contrast to benign E-cadherin-expressing $\operatorname{Tr} 553^{\Delta / \Delta}$ cells, the p120-Mrip interaction was apparent in metastatic E-cadherindeficient mILC cells, providing a rationale for the manifestation of active RhoA in the presence of cytosolic p120 and, furthermore, offering an explanation for the cell type-specific differences regarding the activation of pathways downstream of p120. We anticipate that at least 2 mechanisms could account for the presence of active Rho/Rock signaling in ILC. First, binding of p120 to Mrip may render p120 incapable of exerting its established Rho-modulating function. Unfortunately, we could not verify this option due to severe cell death upon Mrip KD, which could be caused by either strong inhibition of actin dynamics or artificial hyperactivation of the Rock pathway. Second, we rationalize that p120-Mrip interactions may prevent Mrip from inhibiting downstream Rho signals $(20,22,43)$, thus promoting anoikis resistance.

In conclusion, our data established a functional link among early loss of E-cadherin, cytosolic translocation of p120, and subsequent Rock-mediated regulation of anchorage-independent tumor growth of ILC. Using our mouse models of human ILC, we detected active Rho/Rock signaling in the presence of endogenous cytosolic p120 as a consequence of the interaction of cytosolic p120 with Mrip. Since both p120 and Mrip display Rho/Rock-inhibitory functions, we believe this provides an alternative and novel mechanism for the regulation of anoikis resistance in metastatic breast cancer. In the presence of anchorage, the p120-mediated activation of Rho and Rock will be overridden by ECM-mediated integrin crosslinking, which will provide potent outside-in survival signals. Upon the transition to anchorage independence, the integrin-mediated survival signals are lost, and, because cytosolic p120 binds to Mrip, Rock signaling remains active and thus drives anoikis resistance. A schematic working model showing the effects of p120 relocalization, the consequences on downstream Rho/Rock signaling, and the subsequent anoikis resistance is depicted in Figure 7. Our model favors a mechanism whereby during ILC tumor etiology, endogenous p120 has reached an equilibrium with Mrip in such a manner that the net result is an active Rho/Rock signaling pathway that regulates anchorage-independent tumor growth. Because Rock signaling is active in the majority of ILC, and Rock is susceptible to pharmacological inhibition, we reason that the context-dependent manner in which this pathway regulates tumor cell survival provides a window of opportunity for the design of novel clinical intervention strategies. We foresee that inhibition of Rock may be combined with conventional chemotherapy to better treat ILC in the metastatic setting.

\section{Methods}

Cell culture, antibodies, and inhibitors. Mouse mILC- $1\left(\operatorname{Cdh} 1^{\Delta / \Delta} ; \operatorname{Trp} 53^{\Delta / \Delta}\right)$ and $\operatorname{Trp} 53^{\Delta / \Delta}$ cell lines were derived from primary tumors that developed in

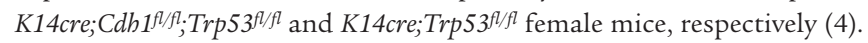
Cell lines mILC-2 and mILC-3 were generated from primary mammary tumors from Wcre;Cdh $1^{f / f l} ; \operatorname{Tr} p 53^{f / f l}$ female mice (25). Cells were cultured as described previously (4). Human breast cancer cell line SKBR-3 (gift of M. Schutte, Erasmus MC, Rotterdam, the Netherlands) was cultured in DMEM/F12 (Invitrogen) containing 6\% fetal calf serum, $100 \mathrm{IU} / \mathrm{ml}$ penicillin, and $100 \mu \mathrm{g} / \mathrm{ml}$ streptomycin. Human primary ILC material was obtained from pleural effusion fluids from a female patient with metastatic ILC. Mononuclear cells were isolated by Ficoll-Paque gradient centrifugation. Purity of the clinical sample exceeded $90 \%$, based on the coexpression of epithelial cytokeratins and ER. Recombinant Wnt3a was provided by R. Nusse (Howard Hughes Medical Institute, Stanford University, Palo Alto, California, USA) The following antibodies were used: mouse anti-p120 (catalog no. 610133; BD Biosciences), TRITC-conjugated p120 (catalog no. 610137; BD Biosciences), mouse anti-E-cadherin (catalog no. 610182; BD Biosciences), mouse anti- $\beta$-catenin (catalog no. 610154 ; BD Biosciences), mouse anti-dephospho- $\beta$-catenin (catalog no. ALX-804-259; Alexis Biochemicals), mouse anti-Rock1 (catalog no. 611136; BD Biosciences), mouse anti-RhoA (catalog no. SC-418; Santa Cruz Biotechnology), goat anti-Akt1 (C-20) (catalog no. SC-1618; Santa Cruz Biotechnology), rabbit anti-Erk1 (C-16) (catalog no. SC-93; Santa Cruz Biotechnology), rabbit anti-phospho-cofilin (Ser3 77g2) (catalog no. 3313; Cell Signaling Technology), rabbit anti-phospho-MLC2 (Ser19) (catalog no. 3671; Cell Signaling Technology), rabbit anti-cofilin (catalog no. 3312; Cell Signaling Technology), rabbit anti-MLC2 (catalog no. 3672; Cell Signaling Technology), mouse anti-HA (12-CA5) (gift from B. Burgering, University Medical Center Utrecht); rabbit anti-HA (HA11 16B12) (catalog no. MMS-101R; Covance), rabbit anti-Mrip (gift from W. Moolenaar, Netherlands Cancer Institute); and Alexa Fluor 488- and Alexa Fluor 633-conjugated phalloidin (both from Invitrogen). Secondary antibodies were Alexa Fluor 488-, Alexa Fluor 561-, and Alexa Fluor 633-conjugated anti-mouse and antirabbit antibodies (Invitrogen). The following inhibitors were used: Y-27632 (10 $\mu \mathrm{M}$ all assays; Sigma-Aldrich), hydroxy fasudil (60 $\mu \mathrm{M}$; Sigma-Aldrich), 
and cell-permeable C3-transferase $(0.02$ and $0.0025 \mu \mathrm{g} / \mathrm{ml}$ for mouse and human cell lines, respectively; Cytoskeleton).

Immunohistochemistry and immunofluorescence. Tissues were isolated and fixed in $4 \%$ formaldehyde for 48 hours. Tissues were dehydrated, cut into 4- $\mu \mathrm{m}$ sections, and stained with H\&E. For single staining, fixed sections were rehydrated and incubated with primary antibodies. Endogenous peroxidases were blocked with $3 \% \mathrm{H}_{2} \mathrm{O}_{2}$ and stained with biotin-conjugated secondary antibodies, followed by incubation with HRP-conjugated streptavidin-biotin complex (DAKO). Substrate was developed with DAB (DAKO). For immunofluorescence, cells were grown on coverslips and fixed using $1 \%$ paraformaldehyde in PBS for 10 minutes. Cells were permeabilized using 0.3\% Triton-X 100 in PBS and subsequently blocked using $5 \%$ BSA (Roche). Coverslips were incubated with primary antibodies in $1 \%$ BSA for 60 minutes, after which cells were incubated in 1\% BSA with secondary antibodies for 30 minutes. DNA was stained with DAPI for 5 minutes (Invitrogen), and coverslips were mounted onto object glasses using Vectashield mounting medium (Vector Laboratories). Cells were analyzed by confocal laser microscopy using a Zeiss LSM510 META (Carl Zeiss).

Constructs, viral production, and transduction. For stable KD of p120, we cloned sequences directed against mouse (5'-GCCAGAAGTGGTGCGAATA-3') and human (5'-GCCAGAGGTGGTTCGGATA-3') p120 that were described previously (26). the Mrip RNAi sequence (5'-GAGCAAGTGTCAGAACTGC- $3^{\prime}$ ) was derived from a previous publication (22). RNAi sequences against mouse Rock1 (sequence 1, 5'-TGTCGAAGATGCCATGTTA-3'; sequence 2, 5'-GACCTTCAAGCACGAATTA-3') were derived from an ON-TARGET SMARTpool (Dharmacon), synthesized as shRNA sequences with BglII/ClaI and XhoI overhangs, and cloned into PSUPER (44). Next, the H1 polymerase promoter-shRNA sequence cassette was removed with enzymes BglII/ClaI and EcoRI and ligated into the lentiviral vector PLVTHM (45). J. Collard (Netherlands Cancer Institute) provided the glutathione-S-transferase-Rho-binding domain (GST-RBD) plasmid. H. Clevers (Hubrecht Institute, Utrecht, the Netherlands) provided the $\beta$-catenin (Ctnnb1) expression plasmid. HA-tagged Mrip was provided in pCDNA3 by W. Moolenaar (19). MluI and NheI sites were added 5' and 3', respectively, by means of Phusion PCR (Thermo Scientific). MluI-HA-MripNheI was subsequently cloned into the lentiviral expression vector pLV. bc.puro (gift from C. Löwik, Leiden University Medical Center, Leiden, the Netherlands). The pCDNA3.IL2R-hEcadTail construct (gift from A. Yap, University of Queensland, Brisbane, Australia) was digested with enzymes EcoRV/XbaI to excise IL-2R-E-cadherin fusion and subsequently cloned into the lentiviral expression vector pLV.bc.puro, yielding pLV.IL2R-hEcadTail.puro (IL2R-Ecad-cyto). p120-1A (Ctnnd1) was released from pEGFPp1201A (gift from J. Daniel, McMaster University, Ontario, Canada) by PCR using Phusion taq polymerase (Thermo Scientific) and cloned into EcoRV-digested pCDNA3.1(+) (Invitrogen). The pmCherry-H2B expression plasmid was a gift from S. Lens (University Medical Center Utrecht). The inducible RNAi system was obtained from TaconicArtemis. Oligos were designed as follows: BbsI-shRNA-XhoI and cloned BbsI/XhoI into the pH1tetflex transfer vector. The H1tetO-shRNA cassette was amplified by means of PCR using Phusion DNA polymerase (Thermo Scientific) and primers with PacI sites. The resulting product was digested with PacI and cloned into FH1tUTG (46). Lentiviral particles were produced in Cos-7 cells using third-generation packaging constructs (47). Supernatant containing viral particles was harvested 48 hours after transfection, passed through a $45-\mu \mathrm{m}$ filter, and concentrated 10 - to 150 -fold by centrifugation $(175,000 \mathrm{~g}$; 150 minutes). mILC cells were transduced overnight in the presence of $4 \mu \mathrm{g} / \mathrm{ml}$ polybrene (Sigma-Aldrich). dox-inducible cell lines were treated for 4 days with $0.1 \mu \mathrm{g} / \mathrm{ml}$ dox, refreshed every 2 days.

Wnt signaling reporter assay. Analysis of canonical Wnt signaling was done using Wnt reporter assays as described previously (48).
Anoikis resistance and colony formation assay. For determination of anoikis resistance, cells were plated and cultured in the absence of EGF and insulin at a density of 100,000 or 20,000 cells/well in a 6- or 24-well ultra-low cluster polystyrene culture dish (Corning), respectively. After 4 days, cells were harvested, and the percentage of anoikis-resistant cells was determined by staining with FITC- or APC-conjugated annexin V (IQ Products) and ToPro-3 (Invitrogen) and analyzed by FACS. Anoikis-resistant cells were defined as the annexin $\mathrm{V}$-ToPro-3- population. For quantification of growth under adherent conditions, cells were plated at a density of 20,000 cells in a 24-well culture plate (Corning) and cultured in the absence of EGF and insulin for 4 days. Cells were subsequently fixed with $100 \%$ methanol, stained using $0.2 \%$ crystal violet (Sigma-Aldrich), and washed with water to remove nonincorporated dye. Incorporated crystal violet was eluted using acetic acid $10 \%(\mathrm{v} / \mathrm{v})$ and quantified with a spectrophotometer at $560 \mathrm{~nm}$ (BioRad).

Rho pulldown, IP, Western blotting, and MS. Cells were lysed in $500 \mu \mathrm{l}$ buffer containing $0.5 \% \mathrm{NP}-40,50 \mathrm{mM}$ Tris $\cdot \mathrm{Cl}$ ( $\mathrm{pH} 7.4$ ), $150 \mathrm{mM} \mathrm{NaCl}, 5 \mathrm{mM}$ $\mathrm{MgCl}_{2}, 10 \%$ glycerol (all Sigma-Aldrich), and protease inhibitors (leupeptin, $10 \mu \mathrm{g} / \mathrm{ml}$; apoptin, $10 \mu \mathrm{g} / \mathrm{ml}$; PMSF, $1 \mu \mathrm{g} / \mathrm{ml}$; Roche). Next, Rho-GTP was isolated from the lysates using the RBD of the human Rhotekin protein (49) fused to GST (residues 7-89) and coupled to gluthatione agarose beads (Sigma-Aldrich). Cells were grown to $80 \%$ confluence, washed twice with calcium-containing PBS, and directly lysed in lysis buffer containing 50 mM Tris-Cl (1M, pH 8.0), 1 mM EDTA, 150 mM NaCl (Riedel-de Haën), $5 \mathrm{mM} \mathrm{NaF}$ (Fluka), 1\% NP40, $2 \mathrm{mM} \mathrm{NaVO}_{3}$, and 1 tablet $/ 50 \mathrm{ml}$ Complete (Roche). Lysates were precleared using protein A/G agarose beads (Millipore). Supernatant was incubated with antibodies for 16 hours at $4^{\circ} \mathrm{C}$, and protein $\mathrm{A} / \mathrm{G}$ beads were added to pull down the complexes. After removal of the unbound fraction, the beads were extensively washed, boiled for 5 minutes in sample buffer, and Western blotted. For phosphorylation assays, cells were lysed directly in buffer containing $50 \mathrm{mM}$ Tris- $\mathrm{Cl}(\mathrm{pH}$ 6.8), $0.5 \% \beta$-mercaptoethanol, $2 \%$ SDS, $0.005 \%$ bromophenolblue, and $10 \%$ glycerol (all Sigma-Aldrich). Samples were heated for 5 minutes at $100^{\circ} \mathrm{C}$, and proteins were separated using standard PAGE protocols and blotting as described previously (50). IP of $\mathrm{p} 120$ protein extracts from mILC- 1 and $\operatorname{Trp} 53^{\Delta / \Delta}$ cells were subjected to SDS-PAGENuPAGE $4 \%-12 \%$ gradient gel (Invitrogen). Protein bands were stained using SYPRO Ruby (Invitrogen) and visualized under UV light. mILC- 1 and $\operatorname{Trp} 53^{\Delta / \Delta}$ lanes were compared, and differentially expressed bands were cut. Peptides were extracted with $10 \%$ formic acid (BDH) and subjected to nanoscale LC-tandem MS analysis, performed on an Agilent 1100 HPLC system (Agilent Technologies) connected to an LTQ Linear Ion Trap Mass Spectrometer combined with an Orbitrap (ThermoFisher) (51). Spectra were searched against the International Protein Index (IPI) mouse database (http://www.ebi.ac.uk/IPI/) using Mascot software (version 2.2.0; Matrix Science). Peptides with mascot scores greater than 35 were considered for further analysis.

Orthotopic transplantations. Recipient female nude mice (Hsd:Athymic Nude-Foxn $1^{\text {nu }}$; Harlan) were anaesthetized with isoflurane (IsoFlo; Le Vet Pharma). The fourth (inguinal) mammary gland was exposed, and approximately 10,000 luciferase-expressing mILC cells were injected using a $10-\mu l$ Hamilton syringe. $100 \mu \mathrm{l}(0.03 \mathrm{mg} / \mathrm{ml})$ Temgesic (buprenorphine) was injected s.c. as analgesic treatment. After a recovery period of 2 weeks, mice were anesthetized with IsoFlo, injected i.p. with $225 \mu \mathrm{g} / \mathrm{g}$ body weight $n$-luciferin (potassium salt; Biosynth AG), and imaged on a Biospace $\Phi$ bioluminescence imager (Biospace Lab). Tumor development was measured using a digital caliper (Mitutoyo) on a weekly basis. Mice were sacrificed when tumor volume exceeded $500 \mathrm{~mm}^{3}$ or when bioluminescence revealed detectable lung metastases.

Statistics. For colony formation and anoikis assays, statistical significance was calculated using 2-tailed Student's $t$ test, showing measurements of 
at least 3 independent experiments. Statistical significance of the genetic inhibition experiments in mice was calculated using the Mann-Whitney test, and error bars represent SEM. Error bars in all other experiments represent SD of at least triplicate measurements. For metastasis-free survival analysis, the log-rank test was used. A $P$ value less than 0.05 was considered statistically significant.

Study approval. All animal experiments were approved by the University Animal Experimental Committee, University Medical Center Utrecht (DEC ABC no. 2007.III.06.081A). Use of anonymous or coded leftover material for scientific purposes is part of the standard treatment contract with patients in our hospitals.

\section{Acknowledgments}

We thank J. Hendriksen, A. Jaspers, and M. Vromans for excellent technical support; members of the Kranenburg, van Rheenen, and Experimental Oncology labs for help and fruitful discussions; W. Moolenaar for the HA-tagged Mrip expression construct and Mrip antibodies; J. Collard for the GST-RBD plasmid; R. Nusse for recombinant Wnt3a; $\mathrm{H}$. Clevers for the $\beta$-catenin expression plasmid; A. Yap for the pCDNA3.IL2R-hEcadTail fusion construct; S. Lens for the pmCherry-H2B construct; J. Daniel for the pEGFP-p1201A construct; the Biomolecular Mass Spectrometry and Proteomics group at Utrecht University for support; and J. de Rooij and D. Pirone for critical review of the manuscript. We are also indebted to the animal facility and the UMC biobank. This work was supported by the UMC Cancer Center, grants from the Netherlands Organization for Scientific Research (NWO-VENI 016.056.135 and NWO-VIDI 917.96.318 to P.W.B. Derksen; NWOVIDI 917.36.347 to J. Jonkers), the Dutch Cancer Society (NKI 2006-3486 to J. Jonkers), and Pink Ribbon.

Received for publication February 7, 2011, and accepted in revised form May 18, 2011.

Address correspondence to: Patrick W.B. Derksen, Department of Pathology, UMC Utrecht, H04.312, Heidelberglaan 100, 3584 CX Utrecht, the Netherlands. Phone: 31.88.7568068; Fax: 31.88.7568479; E-mail: pderksen@umcutrecht.nl.
1. Perez-Moreno M, Jamora C, Fuchs E. Sticky business: orchestrating cellular signals at adherens junctions. Cell. 2003;112(4):535-548.

2. Vos CB, et al. E-cadherin inactivation in lobular carcinoma in situ of the breast: an early event in tumorigenesis. Br J Cancer. 1997;76(9):1131-1133.

3. Berx G, et al. E-cadherin is a tumour/invasion suppressor gene mutated in human lobular breast cancers. EMBO J. 1995;14(24):6107-6115.

4. Derksen PW, et al. Somatic inactivation of E-cadherin and p53 in mice leads to metastatic lobular mammary carcinoma through induction of anoikis resistance and angiogenesis. Cancer Cell. 2006; 10(5):437-449.

5. Shibata T, Kokubu A, Sekine S, Kanai Y, Hirohashi S. Cytoplasmic p120ctn regulates the invasive phenotypes of E-cadherin-deficient breast cancer. Am J Pathol. 2004;164(6):2269-2278.

6. Dillon DA, D'Aquila T, Reynolds AB, Fearon ER, Rimm DL. The expression of $\mathrm{p} 120 \mathrm{ctn}$ protein in breast cancer is independent of alpha- and beta-catenin and E-cadherin. Am J Pathol. 1998;152(1):75-82.

7. Dabbs DJ, Bhargava R, Chivukula M. Lobular versus ductal breast neoplasms: the diagnostic utility of p120 catenin. Am J Surg Pathol. 2007;31(3):427-37.

8. Thoreson MA, Reynolds AB. Altered expression of the catenin 120 in human cancer: implications for tumor progression. Differentiation. 2002;70(9-10):583-589.

9. Anastasiadis PZ, et al. Inhibition of RhoA by p 120 catenin. Nat Cell Biol. 2000;2(9):637-644.

10. Noren NK, Liu BP, Burridge K, Kreft B. p120 catenin regulates the actin cytoskeleton via Rho family GTPases. J Cell Biol. 2000;150(3):567-580.

11. Etienne-Manneville S, Hall A. Rho GTPases in cell biology. Nature. 2002;420(6916):629-635.

12. Kimura K, et al. Regulation of myosin phosphatase by Rho and Rho-associated kinase (Rho-kinase). Science. 1996;273(5272):245-248.

13. Maekawa M, et al. Signaling from Rho to the actin cytoskeleton through protein kinases ROCK and LIM-kinase. Science. 1999;285(5429):895-898

14. Totsukawa G, Yamakita Y, Yamashiro S, Hartshorne DJ, Sasaki Y, Matsumura F. Distinct roles of ROCK (Rho-kinase) and MLCK in spatial regulation of MLC phosphorylation for assembly of stress fibers and focal adhesions in $3 \mathrm{~T} 3$ fibroblasts. J Cell Biol. 2000; 150(4):797-806.

15. Kamai T, et al. Significant association of Rho/ROCK pathway with invasion and metastasis of bladder cancer. Clin Cancer Res. 2003;9(7):2632-2641.

16. Sahai E, Marshall CJ. RHO-GTPases and cancer. Nat Rev Cancer. 2002;2(2):133-142.
17. Zhou J, et al. Gene expression profiles at different stages of human esophageal squamous cell carcinoma. World J Gastroenterol. 2003;9(1):9-15.

18. Kaneko K, Satoh K, Masamune A, Satoh A, Shimosegawa T. Expression of ROCK-1 in human pancreatic cancer: its down-regulation by morpholino oligo antisense can reduce the migration of pancreatic cancer cells in vitro. Pancreas. 2002;24(3):251-257.

19. Gebbink MF, Kranenburg O, Poland M, van Horck FP, Houssa B, Moolenaar WH. Identification of a novel, putative Rho-specific GDP/GTP exchange factor and a RhoA-binding protein: control of neuronal morphology. J Cell Biol. 1997;137(7):1603-1613.

20. Surks HK, Richards CT, Mendelsohn ME. Myosin phosphatase-Rho interacting protein. A new member of the myosin phosphatase complex that directly binds RhoA. J Biol Chem. 2003;278(51):51484-51493.

21. Riddick N, Ohtani K, Surks HK. Targeting by myosin phosphatase-RhoA interacting protein mediates RhoA/ROCK regulation of myosin phosphatase. J Cell Biochem. 2008;103(4):1158-1170.

22. Mulder J, Ariaens A, van den Boomen D, Moolenaar WH. p116Rip targets myosin phosphatase to the actin cytoskeleton and is essential for RhoA/ ROCK-regulated neuritogenesis. Mol Biol Cell. 2004;15(12):5516-5527.

23. de Leeuw WJ, et al. Simultaneous loss of E-cadherin and catenins in invasive lobular breast cancer and lobular carcinoma in situ. J Pathol. 1997;183(4):404-411.

24. van de Wetering M, et al. Mutant E-cadherin breast cancer cells do not display constitutive Wnt signaling. Cancer Res. 2001;61(1):278-284.

25. Derksen PW, et al. Mammary-specific inactivation of E-cadherin and p53 impairs functional gland development and leads to pleomorphic invasive lobular carcinoma in mice. Dis Model Mech. 2011; 4(3):347-358.

26. Davis MA, Ireton RC, Reynolds AB. A core function for $\mathrm{p} 120$-catenin in cadherin turnover. $J$ Cell Biol. 2003;163(3):525-534.

27. Gottardi CJ, Wong E, Gumbiner BM. E-cadherin suppresses cellular transformation by inhibiting beta-catenin signaling in an adhesion-independent manner. J Cell Biol. 2001;153(5):1049-1060.

28. Dohn MR, Brown MV, Reynolds AB. An essential role for p120-catenin in Src- and Rac1-mediated anchorage-independent cell growth.JCell Biol. 2009; 184(3):437-450.

29. Soto E, Yanagisawa M, Marlow LA, Copland JA, Perez EA, Anastasiadis PZ. p120 catenin induces opposing effects on tumor cell growth depending on E-cadherin expression. J Cell Biol. 2008;
183(4):737-749.

30. Sander EE, ten Klooster JP, van Delft S, van der Kammen RA, Collard JG. Rac downregulates Rho activity: reciprocal balance between both GTPases determines cellular morphology and migratory behavior. J Cell Biol. 1999;147(5):1009-1022.

31. Egles C, Shamis Y, Mauney JR, Volloch V, Kaplan DL, Garlick JA. Denatured collagen modulates the phenotype of normal and wounded human skin equivalents. J Invest Dermatol. 2008;128(7):1830-1837.

32. Hein TW, Platts SH, Waitkus-Edwards KR, Kuo L, Mousa SA, Meininger GA. Integrin-binding peptides containing RGD produce coronary arteriolar dilation via cyclooxygenase activation. Am J Physiol Heart Circ Physiol. 2001;281(6):H2378-H2384.

33. Taubenberger AV, Woodruff MA, Bai H, Muller DJ, Hutmacher DW. The effect of unlocking RGD-motifs in collagen I on pre-osteoblast adhesion and differentiation. Biomaterials. 2010;31(10):2827-2835.

34. Sahai E, Marshall CJ. Differing modes of tumour cell invasion have distinct requirements for Rho/ ROCK signalling and extracellular proteolysis. Nat Cell Biol. 2003;5(8):711-719.

35. Liu BP, Burridge K. Vav2 activates Rac1, Cdc42, and RhoA downstream from growth factor receptors but not beta1 integrins. Mol Cell Biol. 2000; 20(19):7160-7169.

36. Lin MT, et al. IL-6 induces AGS gastric cancer cell invasion via activation of the $\mathrm{c}-\mathrm{Src} / \mathrm{RhoA} /$ ROCK signaling pathway. Int J Cancer. 2007; 120(12):2600-2608.

37. He M, et al. Vascular endothelial growth factor C promotes cervical cancer metastasis via up-regulation and activation of RhoA/ROCK- $2 /$ moesin cascade. BMC Cancer. 2010;10:170.

38. Lai JM, Hsieh CL, Chang ZF. Caspase activation during phorbol ester-induced apoptosis requires ROCK-dependent myosin-mediated contraction. J Cell Sci. 2003;116(pt 17):3491-3501.

39. Miñambres R, Guasch RM, Perez-Aragó A, Guerri C. The RhoA/ROCK-I/MLC pathway is involved in the ethanol-induced apoptosis by anoikis in astrocytes. J Cell Sci. 2006;119(pt 2):271-282.

40. Coleman ML, Sahai EA, Yeo M, Bosch M, Dewar A, Olson MF. Membrane blebbing during apoptosis results from caspase-mediated activation of ROCK I. Nat Cell Biol. 2001;3(4):339-345.

41. Sebbagh M, Renvoizé C, Hamelin J, Riché N, Bertoglio J, Bréard J. Caspase-3-mediated cleavage of ROCK I induces MLC phosphorylation and apoptotic membrane blebbing. Nat Cell Biol. 2001; 3(4):346-352. 
42. Croft DR, et al. Actin-myosin-based contraction is responsible for apoptotic nuclear disintegration. J Cell Biol. 2005;168(2):245-255.

43. Koga Y, Ikebe M. p116Rip decreases myosin II phosphorylation by activating myosin light chain phosphatase and by inactivating RhoA.J Biol Chem. 2005;280(6):4983-4991.

44. Brummelkamp TR, Bernards R, Agami R. A system for stable expression of short interfering RNAs in mammalian cells. Science. 2002;296(5567):550-553.

45. Wiznerowicz M, Trono D. Conditional suppression of cellular genes: lentivirus vector-mediated drug-inducible RNA interference. J Virol. 2003;
$77(16): 8957-8961$

46. Herold MJ, van den Brandt J, Seibler J, Reichardt $\mathrm{HM}$. Inducible and reversible gene silencing by stable integration of an shRNA-encoding lentivirus in transgenic rats. Proc Natl Acad Sci U S A. 2008; 105(47):18507-18512.

47. Dull $\mathrm{T}$, et al. A third-generation lentivirus vector with a conditional packaging system. $J$ Virol. 1998;72(11):8463-8471.

48. Hendriksen J, et al. Plasma membrane recruitment of dephosphorylated beta-catenin upon activation of the Wnt pathway. J Cell Sci. 2008; 121(pt 11):1793-1802.
49. Reid T, et al. Rhotekin, a new putative target for Rho bearing homology to a serine/threonine kinase, $\mathrm{PKN}$, and rhophilin in the rho-binding domain. J Biol Chem. 1996;271(23):13556-13560.

50. Derksen PW, et al. Illegitimate WNT signaling promotes proliferation of multiple myeloma cells. Proc Natl Acad Sci U S A. 2004;101(16):6122-6127.

51. Pinkse MW, Mohammed S, Gouw JW, van Breukelen B, Vos HR, Heck AJ. Highly robust, automated, and sensitive online $\mathrm{TiO} 2$-based phosphoproteomics applied to study endogenous phosphorylation in Drosophila melanogaster. J Proteome Res. 2008; 7(2):687-697. 